\title{
EFEITO DA ADIÇÃO DE ÁGUA NA EVOLUÇÃO DA TEMPERATURA NO MIOLO DE AGLOMERADOS DURANTE O CICLO DE PRENSAGEM
}

\author{
Carlos Eduardo Camargo de Albuquerque*, Setsuo Iwakiri** \\ * Eng. Florestal, Dr., Depto. de Engenharia e Tecnologia Florestal, UFPR - camargo@ufpr.br \\ ** Eng. Florestal, Dr., Depto. de Engenharia e Tecnologia Florestal, UFPR - setsuo@floresta.ufpr.br \\ Recebido para publicação: 22/03/2005 - Aceito para publicação: 30/04/2005
}

\begin{abstract}
Resumo
Efeito da adição de água na evolução da temperatura no miolo de aglomerados durante o ciclo de prensagem. Este trabalho analisou a evolução da temperatura no miolo do colchão de partículas, em tempo real, durante o ciclo de prensagem, com e sem o efeito da adição de água nas camadas superficiais do colchão. A adição de água provocou uma acentuada oscilação de temperatura no miolo dos painéis durante o ciclo de prensagem, que requer um tempo total de prensagem superior aos estudados, para atingir uma consolidação efetiva. Sem a adição de água, o tempo total de prensagem de 4 minutos foi suficiente para uma consolidação efetiva dos painéis.

Palavras-chave: aglomerados; ciclo de prensagem; evolução da temperatura.
\end{abstract}

\begin{abstract}
Effect of water application on the temperature development in the mat core during the particleboard pressing cycle. The purpose of the present work was to analyze the temperature development in the mat core, during the pressing cycle, through real time temperature measurement, with and without application of water in the mat superficial layers. The application of water caused temperature oscillation which requires an enlargement of the pressing time to reach as effective consolidation in panels. Without application of water, the pressing time period of 4 minutes is enough to reach an effective consolidation in panels.

Keywords: particleboards; pressing cycle; core temperature development.
\end{abstract}

\section{INTRODUÇÃO}

O aglomerado, apesar dos recentes produtos inseridos no mercado nacional de painéis, como o MDF, possui um mercado em expansão. Portanto, para se manter num mercado cada vez mais exigente e competitivo, há necessidade de uma evolução constante do produto. Desta forma, a precisão do controle do ciclo de prensagem na indústria adquire uma vital importância.

Numa prensagem convencional a quente, de acordo com Maku (1959) citado por Hata et al. (1989), quanto mais elevado o teor de umidade das partículas, menor o tempo para o miolo atingir $100^{\circ} \mathrm{C}$. Entretanto, essa temperatura mantem-se constante pelo tempo necessário, até as partículas reduzirem sua umidade para níveis inferiores a $10 \%$ quando, então, a temperatura volta a subir.

Quando a umidade das camadas superficiais do painel é vaporizada, pelo contato com os pratos aquecidos da prensa, migram para o miolo do painel e, desta forma, a resina reage mais facilmente do que se fosse transferência de calor por condução. Entretanto, a umidade em excesso, ao migrar para as camadas internas do painel, impõe um ciclo de prensagem muito mais longo, a fim de eliminar essa umidade pelas bordas evitando a delaminação com a liberação da pressão e abertura da prensa. Outrossim, ressalva-se que umidade em excesso interfere na reação química de polimerização e condensação, inerentes ao processo de cura da resina (Kelly, 1977).

Heebink et al. (1972), citado por Kelly (1977), afirmaram que 12\% representam o teor de umidade ótimo para um colchão de distribuição uniforme de partículas. Umidades inferiores requerem pressões mais elevadas para consolidar o colchão e podem comprometer ligações interpartículas. 
Contudo, umidades superiores requerem ciclos de prensagem mais longos a fim de permitir que o excesso de umidade seja liberado.

Segundo Moslemi (1974), níveis demasiadamente elevados, ou reduzidos, de umidade na mistura de partículas, resultam em problemas operacionais, produzindo chapas com baixa qualidade. Todavia, o teor de umidade ideal depende de inúmeros fatores, como natureza do processo, tipo e dimensão das partículas, densidade da madeira, tipo de resina, entre outros.

O conteúdo de umidade, então, é um dos mais importantes fatores na manufatura de chapas de partículas. $\mathrm{Na}$ indústria, as partículas de madeira são secas a um teor de umidade entre 3 e $6 \%$ dependendo do tipo e da quantidade da resina (Moslemi, 1974; Kollmann; Kuenzi; Stamm, 1975; Tsoumis, 1991). Após a mistura das partículas secas com a resina, o teor de umidade eleva-se para uma faixa compreendida entre 8 e 14\%, quando se emprega resina uréia-formaldeído (Tsoumis, 1991).

Partículas com teor de umidade elevada causam bolsões de vapor na região central da chapa durante a prensagem (Kollmann, 1975; Tsoumis, 1991). Tal efeito prejudica o desenvolvimento de uma resistência adequada na chapa, especialmente a perpendicular a superfície desta, que se torna muito reduzida. Além disso, partículas úmidas demais necessitam de um período de tempo mais longo na prensagem, o que reduz a capacidade de produção da indústria (Kollmann, 1975).

Outrossim, as partículas nas condições demasiadamente secas ou úmidas, também influenciam o comportamento dos aditivos, e conduz um tempo de prensagem muito longo por diversas razões (Kollmann, 1975).

Mallari et al. (1986), empregando madeira de Shorea sp., verificaram que o conteúdo de umidade ótimo para as propriedades e qualidades de aglomerados, considerando diversas resinas, entre elas a UF, foi de $13 \%$.

Um colchão com umidade elevada, torna as partículas mais flexíveis, independente de sua densidade original. A umidade adicionada ajuda a produzir um colchão mais compressível durante a prensagem à quente. Entretanto, a umidade em excesso no colchão requer um tempo total de prensagem mais longo, devido ao efeito retardante na cura do resina. Esta situação pode provocar a delaminação no miolo e, ocasionalmente, esta pode atingir a superfície do painel, durante a prensagem à quente (Moslemi, 1974).

Em contrapartida, o colchão, com uma umidade excessivamente reduzida, provoca uma baixa transferência de calor da superfície para o miolo. Portanto, o painel obtido pode ter uma densidade não uniforme, além de uma aspereza superficial inaceitável. Além disso, provoca uma inibição do fluxo da resina e transferência, e as partículas muito secas geram uma alta absorção de resina, prejudicando atingir uma liga suficiente na área de contato partícula-partícula. Contudo, o principal inconveniente associado à um colchão muito seco, está relacionado com a redução da área total de contato na estrutura do colchão, devido à falta de flexibilidade, particularmente nas espécies de madeira densa (Moslemi, 1974).

De acordo com Winistorfer (1999), numa prensagem à quente, a transferência de calor e vapor aliada à velocidade de consolidação e características do colchão de partículas, interagem produzindo um gradiente vertical de densidade nos painéis, caracterizado por alta densidade nas camadas superficiais e baixa densidade no miolo.

Segundo Kelly (1977), completado por Suo e Bowyer (1994), o gradiente de densidade é muito dependente da geometria das partículas, distribuição de umidade no colchão, razão de compactação, tempo de fechamento da prensa, temperatura dos pratos da prensa, reatividade da resina, e resistência à compressão das partículas.

Wong et al. (1998), estudando chapas de aglomerados produzidas com partículas de Shorea spp. e isocianato como resina, verificaram que as chapas produzidas com alta umidade nas faces e baixa umidade na parte central, promovem um aumento na densidade máxima existente no gradiente vertical de densidade, em até $22 \%$ e $12 \%$, respectivamente, em chapas de 0,5 e $0,7 \mathrm{~g} / \mathrm{cm}^{3}$ de densidade média.

Segundo Moslemi (1974), é aconselhável que chapas produzidas com madeira de baixa densidade possuam um conteúdo de umidade relativamente inferior àquelas produzidas com madeiras densas. Neste caso, a manufatura de um painel requer um grande volume de partículas, que sofrerá uma compactação, com escape elevado de água na forma de vapor, em um intervalo de tempo menor, pois o ciclo de prensagem requerido é curto. Como a compactação das partículas dificulta a permeabilidade, o 
fluxo de vapor é prejudicado, e uma umidade em excesso no colchão de partículas pode produzir chapas de baixa resistência e, em casos extremos, causar estouros e bolhas nesta chapas.

Sekino (1996) afirmou que a prensagem à quente promove uma redução de $7 \%$ a $27 \%$ na higroscopicidade nos painéis, devido ao aumento na acidez e cristalinidade das partículas submetidas ao calor e pressão. Este efeito também é notado entre as camadas superficiais e o miolo, devido às camadas superficiais receberem temperaturas e pressões mais elevadas.

O presente trabalho objetivou analisar a evolução da temperatura no miolo do colchão de partículas, em tempo real, durante o ciclo de prensagem e, através dos ensaios físicos-mecânicos, os efeitos do tempo de prensagem e da adição de água nas camadas superficiais do colchão de partículas.

\section{MATERIAL E MÉTODOS}

\section{Matéria-prima}

Para o presente estudo, foi estabelecido o emprego de material particulado proveniente da empresa Berneck Aglomerados S.A., que forneceu as partículas prontas para utilização, assim como a resina tipo uréia-formaldeído (UF) e catalisador.

\section{Preparação da matéria-prima}

A matéria-prima da referida empresa, Pinus sp., consiste em toretes oriundos de fazendas fornecedoras, resíduos de serraria própria e de terceiros, e maravalha de terceiros. Para o presente estudo, o material particulado empregado consistiu na matéria-prima processada obtida diretamente da linha de produção na saída dos secadores rotativos. Outrossim, empregou-se um tipo de geometria de partículas: destinada a camada interna dos painéis, caracterizada pela predominância de partículas grossas.

O material coletado foi acondicionado em sacos plásticos, devidamente identificados, e em seguida conduzido ao laboratório para a manufatura dos painéis e dos posteriores testes.

\section{Formação do colchão}

As partículas foram misturadas com a resina por meio de pulverização, em um aplicador tipo tambor rotativo e, então, foram depositadas manualmente em uma caixa de formação nas dimensões de $50 \times 55 \mathrm{~cm}$. Utilizou-se placas de alumínio nas superfícies para evitar o contato direto do colchão com os pratos quentes da prensa.

\section{Uso de termopares}

O presente trabalho baseou-se no desenvolvimento da temperatura no interior dos painéis durante a prensagem à quente. Portanto, instalaram-se cabos termopares do tipo "J" no centro de cada colchão de partículas (Figura 1) antes da prensagem, o qual ligava-se à um módulo condicionador de sinais analógicos acoplado à uma placa de conversão analógico/digital instalada em um microcomputador, onde os dados gerados foram armazenados e processados. As leituras de temperaturas foram realizadas em intervalos de 1 segundo durante o período de prensagem (Figura 2).

A colocação dos termopares foi realizada durante a formação do colchão de partículas já misturadas com a resina. Ou seja, após a pesagem, depositou-se $50 \%$ da mistura partículas-resina na caixa de formação e, então, posicionou-se o termopar no centro da massa de partículas, terminando-se esta operação com a distribuição do restante da mistura. 


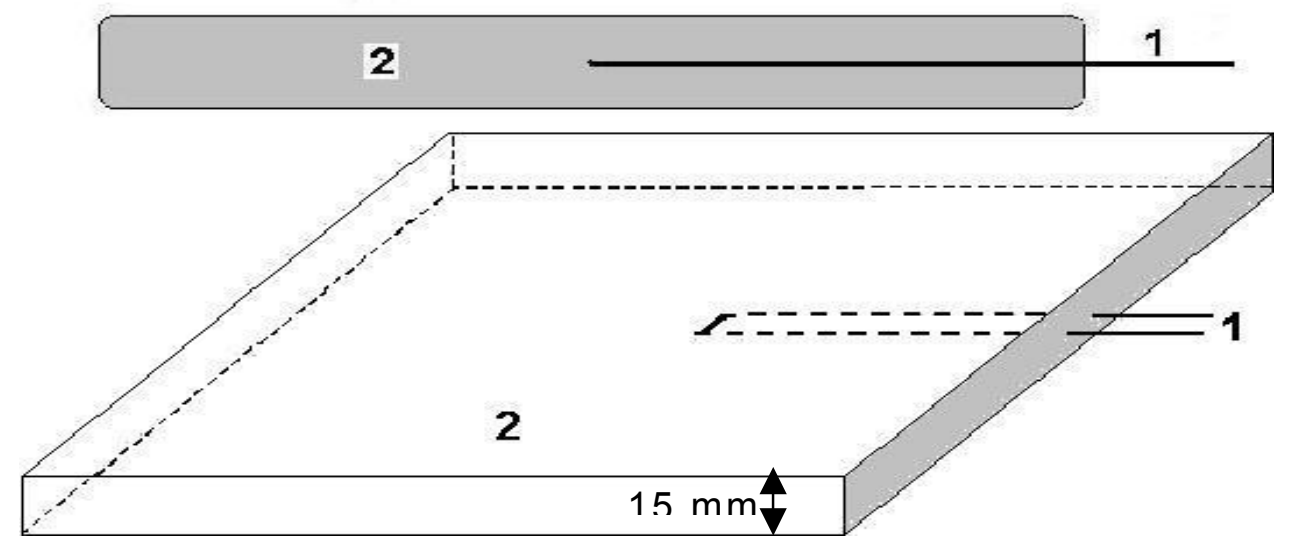

Figura 1. Esquema do posicionamento do cabo termopar no colchão de partículas antes da prensagem, sendo:

1. cabo termopar posicionado a $1 / 2$ da espessura do colchão;

2. colchão de partículas;

Figure 1. Sketch of thermocouple cable posicioned in the mat core:

1. thermocouple cable posicioned at the middle of mat thickness;

2. mat construction.

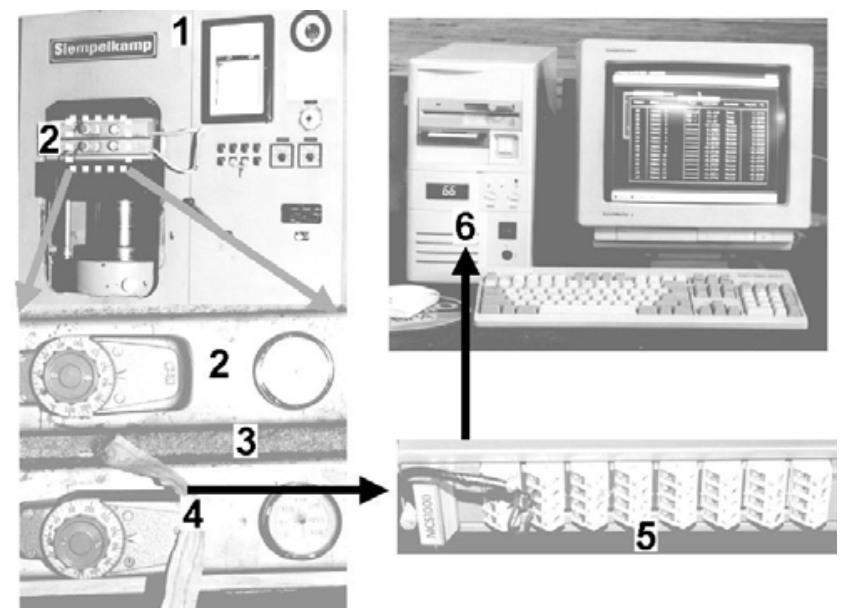

Figura 2. Equipamento montado em funcionamento:

1. prensa hidráulica; 2. pratos eletricamente aquecidos; 3. colchão de partículas;

4. cabo termopar; 5. módulo condicionador de sinais analógicos; 6. microcomputador.

Figure 2. Working assembled equipment:

1. hidraulic press; 2. electric hot plates; 3. mat; 4. thermocouple cable; 5. condicioner module of analogic signals; 6 . microcomputer.

\section{Prensagem}

\section{Pré-definição das condições fixas de prensagem}

Com a conclusão da etapa de formação do colchão, este foi conduzido à prensa, onde efetivou-se a prensagem, sob $40 \mathrm{kgf} / \mathrm{cm}^{2}$ de pressão específica e $150^{\circ} \mathrm{C}$ de temperatura.

Outrossim, como se objetivou o controle da temperatura no interior dos painéis, o início destas medições deu-se no momento em que as duas faces do colchão entravam em contato com os pratos 
aquecidos da prensa, situação esta que ocorria no início do fechamento da prensa. Este posicionamento se baseou no fato de que o início da transmissão da temperatura, dos pratos da prensa para o colchão de partículas, iniciava-se no supracitado momento.

O tempo de fechamento da prensa, por sua vez, não foi considerado como variável neste estudo, mas correspondeu ao valor aproximado de 50 segundos.

\section{Tempo de prensagem}

O tempo de prensagem foi uma variável experimental considerada, mas não da forma convencional. O presente estudo baseou-se no tempo transcorrido até que o miolo do painel atingisse $95^{\circ} \mathrm{C}$, mais os adicionais de tempo de 0,1 e $2 \mathrm{~min}$.

Após a conclusão da prensagem, os painéis foram identificados, esquadrejados e, então, conduzidos à câmara de climatização sob $20 \pm 3^{\circ} \mathrm{C}$ de temperatura e $65 \%$ de umidade relativa do ar.

\section{Avaliação dos parâmetros de estudo: temperatura, densidade e espessura}

Analisou-se a evolução da temperatura no miolo dos painéis, durante o ciclo de prensagem, assim como a densidade e a espessura final dos mesmos.

\section{Considerações sobre o plano experimental}

Neste estudo, optou-se pelo ciclo de prensagem em que o parâmetro base do experimento fosse a temperatura de $95^{\circ} \mathrm{C}$ a ser atingida no miolo do painel. Portanto, deu-se início a fase experimental considerando-se as seguintes variáveis:

1. material particulado grosso, utilizado como camada interna de aglomerados pela indústria;

2. densidade para os painéis de $0,7 \mathrm{~g} / \mathrm{cm}^{3}$ e nível de resina de $8 \%$;

3. 3 umidades para o colchão de partículas - com adição, por pulverização, de $0 \%, 3 \%$ e $6 \%$ de umidade nas camadas superficiais do colchão, antes da prensagem. Cada camada corresponde, em peso, a 1/4 do painel.

O delineamento experimental seguiu o apresentado na Tabela 1.

Os resultados obtidos foram analisados pela aplicação de testes de correlação e por comparações entre médias pelo teste de Tukey, adotando-se o nível de significância de $5 \%$ de probabilidade. Outrossim, foram analisadas as interações entre as variáveis estudadas.

Tabela 1. Delineamento experimental.

Table 1. Experimental outline.

\begin{tabular}{l|c}
\multicolumn{1}{c|}{ TEMPO DE PRENSAGEM } & $\begin{array}{c}\text { ÁGUA ADICIONADA } \\
(\%)\end{array}$ \\
\hline até miolo atingir $95^{\circ} \mathrm{C}$ & 0 \\
1 min após miolo atingir $95^{\circ} \mathrm{C}$ & 0 \\
2 min após miolo atingir $95^{\circ} \mathrm{C}$ & 0 \\
1 min após miolo atingir $95^{\circ} \mathrm{C}$ & 3 \\
2 min após miolo atingir $95^{\circ} \mathrm{C}$ & 3 \\
1 min após miolo atingir $95^{\circ} \mathrm{C}$ & 6 \\
2 min após miolo atingir $95^{\circ} \mathrm{C}$ & 6 \\
\hline
\end{tabular}

\section{RESULTADOS E DISCUSSÃO}

\section{Evolução da temperatura em função do tempo}

A evolução da temperatura durante a prensagem nos tratamentos estudados se apresentou, de modo geral, numa forma progressiva e regular, com temperatura máxima de $118,4^{\circ} \mathrm{C}$ (Figura 3 ). Contudo, os tratamentos que empregaram a adição de 3\% de água nas camadas superficiais do colchão de partículas, apresentaram uma oscilação da temperatura durante sua evolução (Figura 4), apresentando temperatura máxima de $116,1^{\circ} \mathrm{C}$, ligeiramente inferior ao tratamento sem adição de água. No caso dos tratamentos que aplicaram $6 \%$ de água, a oscilação foi ainda mais acentuada, com temperatura máxima registrada de $113,7^{\circ} \mathrm{C}$, inferior aos casos anteriores (Figura 5). Verificou-se, desta forma, que a adição de água ocasionou uma acentuada oscilação de temperatura na fase de aquecimento do colchão, agravando- 
se quando do aumento da quantidade de água de 3\% para 6\%, além de proporcionar uma ligeira redução nas temperaturas máximas registradas, provavelmente devido ao excesso de água ainda presente no colchão no final da prensagem.

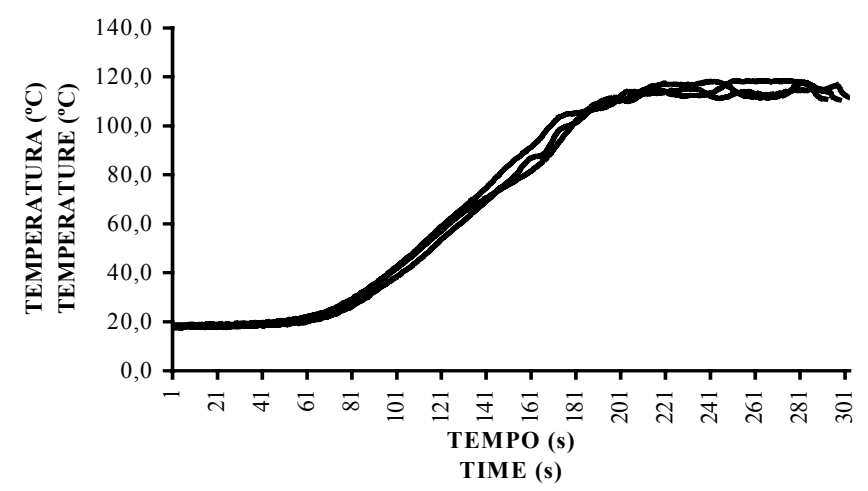

Figura 3. Evolução da temperatura até 2 min após o miolo do painel atingir $95^{\circ} \mathrm{C}\left(\mathrm{T}^{\circ}\right.$ máx $\left.=118,4^{\circ} \mathrm{C}\right)$; tratamento: $0 \%$ de adição de água ( 3 repetições).

Figure 3. Temperature development up to 2 min after to be reached $95^{\circ} \mathrm{C}$ (peak $\mathrm{T}^{\mathrm{o}}=118,4^{\circ} \mathrm{C}$ ) in the mat core; treatment: $0 \%$ water application (3 repetitions).

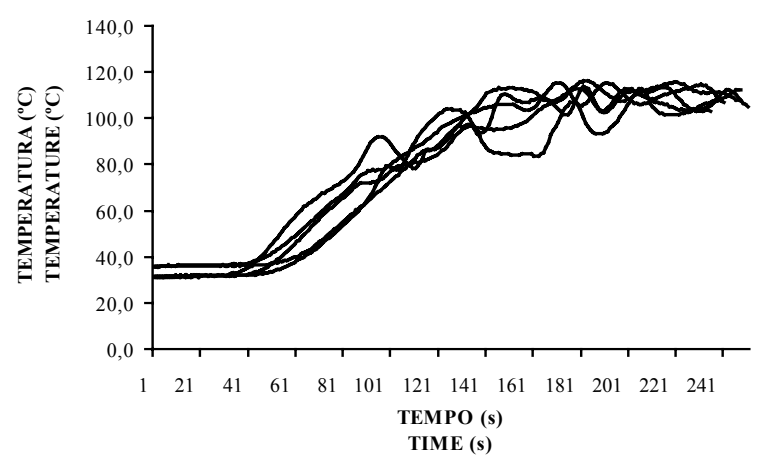

Figura 4. Evolução da temperaura até 2 min após o miolo do painel atingir $95^{\circ} \mathrm{C}$ ( $\mathrm{T}^{\circ}$ máx: $116,1^{\circ} \mathrm{C}$ ); tratamento: adição de 3\% de água (5 repetições).

Figure 4. Temperature development up to 2 min after to be reached $95^{\circ} \mathrm{C}$ (peak $\mathrm{T}^{\mathrm{o}}: 116,1^{\circ} \mathrm{C}$ ) in the mat core; treatment: $3 \%$ water application (5 repetitions). 


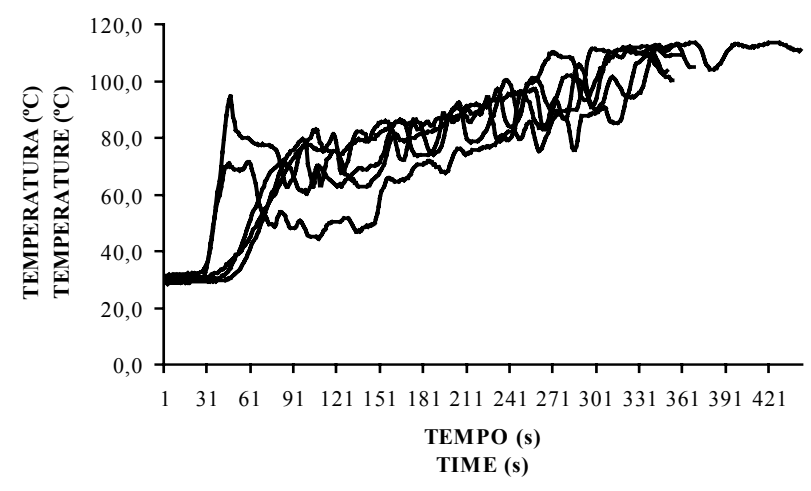

Figura 5. Evolução da temperatura até 2 min após o miolo do painel atingir $95^{\circ} \mathrm{C}\left(\mathrm{T}^{\circ}\right.$ máx $\left.=113,7^{\circ} \mathrm{C}\right)$; tratamento: adição de $6 \%$ de água (5 repetições).

Figura 5. Temperature development up to $2 \mathrm{~min}$ after to be reached $95^{\circ} \mathrm{C}$ (peak $\mathrm{T}^{\mathrm{o}}=113,7^{\circ} \mathrm{C}$ ) in the mat core; treatment: $6 \%$ water application (5 repetitions).

A partir da análise das médias dos tempos transcorridos para se atingir $95^{\circ} \mathrm{C}$ no miolo dos painéis (Tabela 2), observou-se algumas situações.

Tabela 2. Efeito da adição de água no tempo de aquecimento no miolo do painel até atingir $95^{\circ} \mathrm{C}$.

Table 2. Added water effect on heating time up to $95^{\circ} \mathrm{C}$ to be reached in panel core.

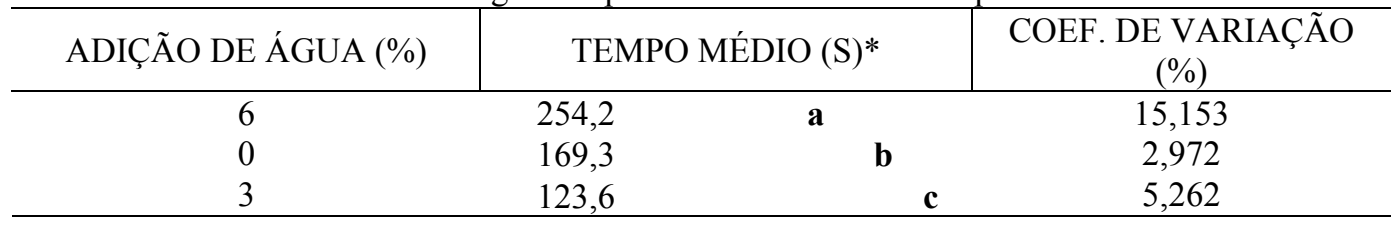

* Médias seguidas da mesma letra não diferem estatisticamente entre si, ao nível de $95 \%$ de probabilidade, pelo teste de Tukey. Averages followed by the same letter are not different at the statistic point of view (Tukey 95\%).

\section{Efeito da adição de água no tempo de aquecimento do painel}

Observou-se uma redução estatisticamente significativa no tempo para se atingir $95^{\circ} \mathrm{C}$ nos tratamentos em que se aplicaram 3\% de umidade nas camadas superficiais do colchão, ou seja, a elevação inicial de temperatura foi mais acelerada e suficiente para atingir $95^{\circ} \mathrm{C}$.

Verificou-se, contudo, uma oscilação na evolução da temperatura acima dos $95^{\circ} \mathrm{C}$, não verificada nos tratamentos sem adição de água, ou seja, a transmissão de calor foi irregular. Esta oscilação, entretanto, ocorreu em temperaturas próximas a $100^{\circ} \mathrm{C}$ e, segundo Strickler (1959), durante o fluxo de vapor, pode ocorrer uma condensação do vapor nas camadas centrais mais frias do painel. Além disso, após o aumento inicial de temperatura no miolo, há uma perda de calor, provavelmente, devido a perda de umidade na camada interna, assim como ao calor consumido na evaporação da água para a atmosfera através da linha central, e nem tanto pela liberação através da condensação da água que vem da superfície na forma de vapor (Maku et al., 1959; Strickler, 1959 citados por Kelly, 1977).

Portanto, a rápida redução do fluxo de vapor vindo da superfície, combinado com a condensação e troca de calor com as partículas e a perda da umidade da camada interna pelas bordas do painel, explicaria esta oscilação de temperatura. Ressalva-se ainda que, parte da energia dos pratos quentes foi absorvida na vaporização da umidade aplicada, assim como na umidade presente nas partículas. Aliado a isto, o fato da quantidade de água aplicada nas superfícies (3\%) ter sido relativamente pequena, permitiu seu rápido aquecimento e vaporização para o interior do painel, esgotando-se logo das camadas 
superficiais, permitindo o aumento da temperatura nestas camadas para valores acima de $100^{\circ} \mathrm{C}$, que rapidamente se transmitiu para o miolo.

No caso dos tratamentos com adição de umidade, o fluxo de vapor não é contínuo e nem homogêneo e, com a adição de $6 \%$ de umidade, em especial, a temperatura do miolo se elevou rapidamente nos momentos iniciais da prensagem, mas diferentemente das afirmações de Maku (1959) citado por Hata et al. (1989), não atingiu rapidamente os $100^{\circ} \mathrm{C}$, pois antes de se atingir $95^{\circ} \mathrm{C}$, iniciou-se uma oscilação acentuada da mesma, demonstrando uma transmissão de calor bastante irregular. Quanto ao tempo para se atingir $95^{\circ} \mathrm{C}$, neste caso, foi estatisticamente superior aos tratamentos sem adição de água e aos que aplicaram $3 \%$ de água. Ou seja, o aumento da umidade aplicada nas camadas superficiais de $3 \%$ para $6 \%$ aumentou o tempo para se atingir $95^{\circ} \mathrm{C}$. Neste caso, a quantidade de água aplicada nas superfícies de 6\% consumiu mais energia dos pratos da prensa, que segundo Strickler (1959), no início da prensagem, a rápida transferência de calor para a superfície do colchão provoca uma momentânea redução de temperatura dos pratos aquecidos por 1 ou 2 min. Com a demora da eliminação do excesso de água pelas bordas, a temperatura não se eleva acima $\operatorname{dos} 100^{\circ} \mathrm{C}$, retardando o aquecimento das camadas superficiais e, por conseguinte, do painel, assim como a oscilação de temperatura.

Em resumo, diferentemente do encontrado na literatura, um aumento na quantidade de umidade aplicada nas camadas superficiais, dependendo da quantidade, pode aumentar o tempo para se atingir $100^{\circ} \mathrm{C}$ no miolo, embora o início da prensagem apresente um crescimento muito rápido da temperatura. Além disso, ocorre uma oscilação muito acentuada e demorada da temperatura no miolo, tornando um processo de aquecimento muito irregular e de difícil controle. Ou seja, os resultados obtidos mostram que a adição de umidade nas camadas superficiais deve ser efetuada com quantidades reduzidas de umidade já que o objetivo seria de aumentar o gradiente vertical de densidade a fim de obter melhores resultados em flexão estática.

As Tabelas 3 e 4 apresentam o resultado do teste de Tukey para densidade e espessura. Os painéis produzidos com o tempo de prensagem definido pelo período de, até o miolo atingir $95^{\circ} \mathrm{C}$, tiveram suas densidades significativamente abaixo do esperado, enquanto que os painéis produzidos com acréscimo de dois minutos, acima dos anteriores, obtiveram densidade compatível com o esperado. Portanto, houve consolidação mais efetiva neste caso, enquanto que os tempos de prensagem inferiores proporcionaram um retorno em espessura decorrente da liberação das tensões de compressão nos painéis após a finalização da prensagem, provavelmente devido à consolidação não efetiva, reduzindo a densidade. Este fato pode ser comprovado na Tabela 4, onde se verificam aumentos significativos em espessura para tempos menores de prensagem.

Tabela 3. Efeito do tempo de prensagem na densidade dos painéis programados para $0,7 \mathrm{~g} / \mathrm{cm}^{3}$.

Table 3. Pressing time effect on particleboard density designed to $0,7 \mathrm{~g} / \mathrm{cm}^{3}$.

\begin{tabular}{l|cc|c}
$\begin{array}{c}\text { TEMPO DE } \\
\text { PRENSAGEM }\end{array}$ & DENSIDADE MÉDIA $\left(\mathrm{g} / \mathrm{cm}^{3}\right)$ & COEF. DE VARIAÇÃO (\%) \\
\hline 2 min após $95^{\circ} \mathrm{C}$ & $0,69 \quad \mathbf{a}$ & 3,98 \\
\hline 1 min após $95^{\circ} \mathrm{C}$ & 0,61 & b & 3,64 \\
\hline até $95^{\circ} \mathrm{C}$ & 0,58 & c & 3,00 \\
\hline
\end{tabular}

* Médias seguidas da mesma letra não diferem estatisticamente entre si, ao nível de 95\% de probabilidade, pelo teste de Tukey. Averages followed by the same letter are not different at the statistic point of view (Tukey 95\%). 
Tabela 4. Efeito do tempo de prensagem na espessura do painel.

Table 4. Pressing time effect on panel thickness.

\begin{tabular}{l|rr|c}
\hline $\begin{array}{c}\text { TEMPO DE } \\
\text { PRENSAGEM }\end{array}$ & \multicolumn{2}{|c|}{ ESPESSURA MÉDIA $(\mathrm{mm})$} & COEF. DE VARIAÇÃO (\%) \\
\hline até $95^{\circ} \mathrm{C}$ & $17,1 \quad \mathbf{a}$ & $\mathbf{b}$ & 0,80 \\
\hline $95^{\circ} \mathrm{C}+1 \mathrm{~min}$ & 16,3 & c & 1,70 \\
\hline $95^{\circ} \mathrm{C}+2 \mathrm{~min}$ & 14,4 & 1,08 \\
\hline
\end{tabular}

* Médias seguidas da mesma letra não diferem estatisticamente entre si, ao nível de $95 \%$ de probabilidade, pelo teste de Tukey. Averages followed by the same letter are not different at the statistic point of view (Tukey 95\%).

\section{Influência do tempo de prensagem}

Diferentemente dos procedimentos tradicionais, o tempo de prensagem aplicado neste estudo não é baseado em períodos de tempo fixos, e sim, de uma condição preestabelecida a ser atingida, mais especificamente, a partir do tempo necessário para que o miolo do painel atinja $95^{\circ} \mathrm{C}$. Desta forma, acredita-se estar analisando, de forma mais fiel, os resultados decorrentes da prensagem, visto que tratase de um processo que emprega uma matéria-prima muito heterogênea, onde inúmeras variáveis interferem e se interagem e, por conseguinte, não seria possível a determinação de um tempo fixo ideal de prensagem, pois acredita-se que o tempo é ideal para cada painel, e para conseguí-lo, torna-se necessário a definição de uma condição base a ser atingida em cada ciclo de prensagem.

Desta forma, pode-se concluir que houve início do processo de cura da resina no miolo dos painéis, mesmo naqueles onde o tempo de prensagem cessou ao se atingir $95^{\circ} \mathrm{C}$ nesta região. Ademais, por alguns momentos, após a abertura da prensa, a energia já absorvida pelos painéis foi suficiente para que o miolo aumentasse sua temperatura por alguns graus, geralmente atingindo temperaturas em torno de $100^{\circ} \mathrm{C}$ (Tabela 5), o que reforça o descrito na literatura, ou seja, houve temperatura suficiente para início do processo de cura da resina no miolo do painel.

Tabela 5. Média das temperaturas alcançadas no miolo do painel depois de 4,6 e 8 segundos após se atingir $95^{\circ} \mathrm{C}$ (término da prensagem).

Table 5. Average temperatures in the panel core up to 4,6 and 8 seconds after $95^{\circ} \mathrm{C}$ to be reached (end cycle press).

\begin{tabular}{|c|c|c|c|c|}
\hline \multirow{2}{*}{ TRATAMENTO } & \multicolumn{4}{|c|}{ TEMPERATURA $\left({ }^{\circ} \mathrm{C}\right)$ APÓS TÉRMINO DA PRENSAGEM } \\
\hline & TÉRMINO & TÉRMINO + 4s & TÉRMINO + 6s & TÉRMINO + 8s \\
\hline $\begin{array}{l}\text { TEMPO DE PRENSAGEM ATÉ } \\
\text { O MIOLO DO COLCHÃO } \\
\text { ATINGIR } 95^{\circ} \mathrm{C}, \quad \text { SEM } \\
\text { APLICAÇÃO DE ÁGUA }\end{array}$ & 95 & 98,2 & 99,3 & 100,5 \\
\hline
\end{tabular}

As Tabelas 6 e 7 apresentam, respectivamente, a alteração da densidade e da espessura dos painéis, conforme a adição de água, considerando os tempos de prensagem de 1 e 2 min após atingir $95^{\circ} \mathrm{C}$ no miolo do painel. Considerando o tempo de $1 \mathrm{~min}$ após se atingir $95^{\circ} \mathrm{C}$, não houve diferenças estatísticas, evidenciando tempo de prensagem insuficiente para uma consolidação mais efetiva. Contudo, aumentando-se o tempo de prensagem para $2 \mathrm{~min}$ após se atingir $95^{\circ} \mathrm{C}$, observa-se que há um aumento estatístico na densidade dos painéis que não receberam água, e uma igualdade estatística entre os que receberam 3 e 6\% de água, evidenciando uma consolidação mais efetiva nos painéis que não receberam água.

$\mathrm{O}$ aumento em espessura verificado nos painéis com prensagem até $95^{\circ} \mathrm{C}$ (Tabela 4) ocorreu logo após a abertura da prensa, reduzindo a densidade do painel e elevando a porosidade. Ou seja, a massa lenhosa reagiu liberando as tensões de compressão decorrente da consolidação não efetiva. 
Tabela 6. Efeito da adição de água na densidade, para o tempo de prensagem de 1 e 2 min após atingir $95^{\circ} \mathrm{C}$ no miolo do painel.

Table 6. Added water effect on density, to pressing time up to 1 and 2 minutes after $95^{\circ} \mathrm{C}$ to be reached in panel core.

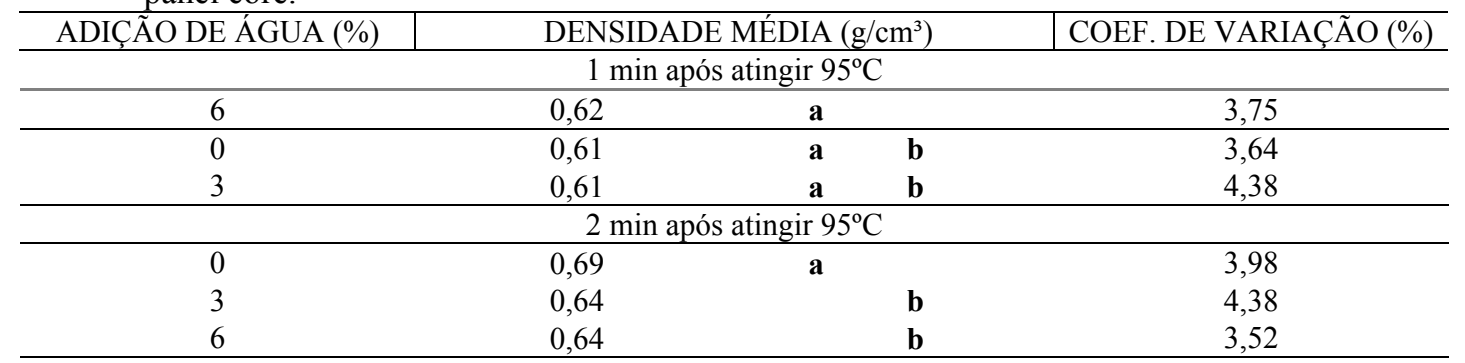

* Médias seguidas da mesma letra não diferem estatisticamente entre si, ao nível de 95\% de probabilidade, pelo teste de Tukey. Averages followed by the same letter are not different at the statistic point of view (Tukey 95\%).

Tabela 7. Efeito da adição de água na espessura do painel para o tempo de prensagem de 1 e 2 min após atingir $95^{\circ} \mathrm{C}$ no miolo.

Table 7. Added water effect on panel thickness, to pressing time up to 1 and 2 minutes after $95^{\circ} \mathrm{C}$ to be reached in panel core.

\begin{tabular}{|c|c|c|}
\hline $\begin{array}{c}\text { ADIÇÃO DE } \\
\text { ÁGUA }(\%)\end{array}$ & ESPESSURA MÉDIA (mm) & COEF. DE VARIAÇÃO (\%) \\
\hline \multicolumn{3}{|c|}{$1 \mathrm{~min}$ após atingir $95^{\circ} \mathrm{C}$} \\
\hline 3 & 16,5 & 2,41 \\
\hline 0 & 16,3 & 1,70 \\
\hline 6 & 16,1 & 2,84 \\
\hline \multicolumn{3}{|c|}{2 min após atingir $95^{\circ} \mathrm{C}$} \\
\hline 3 & 16,0 & 1,33 \\
\hline 6 & 15,8 & 2,98 \\
\hline 0 & 14,4 & 1,08 \\
\hline
\end{tabular}

* Médias seguidas da mesma letra não diferem estatisticamente entre si, ao nível de $95 \%$ de probabilidade, pelo teste de Tukey. Averages followed by the same letter are not different at the statistic point of view (Tukey 95\%).

\section{Influência da adição de água associado ao tempo de prensagem}

Neste procedimento, foi abandonado o tempo mais curto de prensagem, ou seja, até o miolo do painel atingir $95^{\circ} \mathrm{C}$. Nesta condição, a adição de água impediu a consolidação do painel, o qual sofreu delaminação imediatamente após a abertura da prensa.

\section{CONCLUSÕES}

1. Tempo de prensagem para o miolo do painel atingir $95^{\circ} \mathrm{C}$ foi insuficiente para uma consolidação efetiva nos painéis que não receberam água, confirmado pelos resultados em densidade, tendo em vista que os resultados obtidos dentro do esperado só foi atingido com o acréscimo de dois minutos após o miolo atingir $95^{\circ} \mathrm{C}$;

2. Tempo de prensagem inferior ao mínimo necessário para uma efetiva consolidação resulta em painéis menos densos, devido ao aumento da espessura destes após a finalização da prensagem, decorrente de uma liberação da forças de compressão;

3. O período de tempo de prensagem, baseado numa condição preestabelecida de temperatura no miolo do painel, permitirá um melhor controle na consolidação do painel, pois será possível determinar e efetuar, simultaneamente, e em tempo real, um período de tempo ideal para a prensagem em curso; 
4. O tempo total de prensagem a partir de 4 minutos, em laboratório, foi suficiente para uma consolidação efetiva. Qualquer condição que aumente o conteúdo de água requer aumento no tempo de prensagem;

5. A adição de $3 \%$ de água nas camadas superficiais do colchão acelera a elevação de temperatura no miolo dos painéis, mas essa evolução se apresenta de forma oscilante;

6. A adição de $6 \%$ de água nas camadas superficiais do colchão torna o aquecimento mais lento nos painéis, apresentando uma oscilação acentuada na evolução da temperatura, evidenciando uma excessiva quantidade de água;

7. A adição de $3 \%$ e $6 \%$ de água requer um tempo total de prensagem mais prolongado, e a oscilação acentuada de temperatura verificada durante o aquecimento dificulta um controle preciso do ciclo de prensagem, prejudicando a determinação de um tempo ideal de prensagem.

8. A associação de adição de água com o tempo mais curto de prensagem, ou seja, até o miolo do painel atingir $95^{\circ} \mathrm{C}$, impediu a consolidação do painel, o qual sofreu delaminação imediatamente após a abertura da prensa.

\section{REFERÊNCIAS}

HATA, T.; SUBIYANTO, B.; KAWAI, S.; SASAKI, H. Production of particleboard with steaminjection. Wood Science and Technology, Berlin, n. 23, p. 361-369. 1989.

KELLY, M. W. Critical literature review of relationships between processing parameters and physical properties of particleboard. Madison: USDA Forest Service General, 1977. 66p. (Technical Report FPL - 10).

KOLLMANN, F. F. P.; KUENZI, E. W.; STAMM, A. J. Principles of wood science and technology. Berlin: Springer-Verlag, 1975.

MALLARI, V. C. JR.; KAWAI, S.; SASAKI, H.; SUBIYANTO, B.; SAKUNO, T. The manufacturing of particleboard I. Types of adhesives and optimum moisture content. Journal of the Japan Wood Research Society, Tokyo, v. 32, n. 6, p. 425-431. 1986.

MOSLEMI, A. A. Particleboard. Illinois: Southern Illinois University Press, 1974.

SEKINO, N., IRLE, M. A. Particleboard hygroscopicity: its prediction and the influence of hot-pressing. Journal of the Japan Wood Research Society, Tokyo, v. 42, n. 1, p. 43-50. 1996.

SUO, S.; BOWYER, J. L. Simulation modeling of particleboard density profile. Wood and Fiber Science, Madison, v. 26, n. 3, p. 397-411. 1994.

TSOUMIS, G. T. Science and technology of wood: structure, properties, utilization. New York: Chapman \& Hall, 1991.

WINISTORFER, P. N.; WANG, S.; IRLE, M.; MATTHEWS, B.; EVANS, A.; PITEMAM, A.; COMBEN, A. Densification of wood composite mats during pressing: implications of mat structure and pressing schedules on density profile formation and panel properties. In: INTERNATIONAL CONFERENCE ON THE DEVELOPMENT OF WOOD SCIENCE, 4., 1999, Missenden Abbey. Proceedings of the Fourth International Conference on the Development of Wood Science, High Wycombe: FPRC, p. 375-382. 1999.

WONG, E. D.; ZHANG-MIN; WANG-QIAN; KAWAI, S. Effects of mat moisture content and press closing speed on the formation of density profile and properties of particleboard. Japan Wood Science, Tokyo, v. 44, p. 287-295. 1998. 